\title{
Belimumab efficacy for the treatment of antibody mediated rejection after heart transplantation: Case report
}

\author{
Mathias Ade ${ }^{1}$, Philippe Billaud ${ }^{2}$ and Eric Epailly ${ }^{2 *}$ \\ ${ }^{1}$ Pharmacy Department, University Hospital Strasbourg, France \\ ${ }^{2}$ Cardiovascular Surgery Department, University Hospital Strasbourg, France
}

\begin{abstract}
Antibody Mediated Rejection (AMR) is a severe complication after heart transplantation (HTx) due to the production of Donor Specific Antibodies (DSA) by the B-lymphocytes (BL) of the recipient. AMR is associated with a high risk of death due to the lack of efficient treatment. We describe the case of a 50-year old patient presenting a pAMR2 graded endomyocardial biopsy (EMB) 22 months post transplantation associated with a NYHA class II dyspnea, a drop of LVEF from $70 \%$ to $40 \%$ and a high MFI DQ5 DSA. Because the EMB showed persistant AMR despite the treatment associating 13 plasma exchange procedures and IVIG treatment followed by a single $375 \mathrm{mg} / \mathrm{m}^{2}$ dose of Rituximab, we decided to introduce Belimumab at a dose of $10 \mathrm{mg} / \mathrm{kg}$ IV at D0, D14 and D2 1 associated to plasma exchanges allowing to normalize the complete regression of AMR on the EMB. Belimumab inhibits the BL survival and their transformation in plasmocytes by linking the BAFF soluble protein and lowering the level of circulating antibodies. This is the first case of a successful Belimumab treatment for a persistent AMR in a HTx patient.
\end{abstract}

\section{Introduction}

Antibody Mediated Rejection (AMR) is a severe complication after heart transplantation (HTx) due to the production of Donor Specific Antibodies (DSA) by the B-lymphocytes (BL) of the recipient which alters the microvascularisation of the graft. The diagnosis is only based on a difficult pathology finding on the endomyocardial biopsy (EMB). AMR is associated with a high risk of death due to the lack of efficient treatment $[1,2]$. This article describes the case of a HTx patient suffering from AMR who was successfully treated with Belimumab.

\section{Clinical case}

A 50-year old patient was heart transplanted in July 2010 due to a cardiogenic shock after LAD coronary vessel occlusion. Pre-transplant Luminex antibody screening and post-transplant LCT crossmatch were negative. Early post-transplant treatment associated Thymoglobulin, Cyclosporine, Mycophenolate Mofetyl and Steroids. Post-transplant systematically EMB were negative and repeated cardiac ultrasound imaging were normal. In April 2011, the patient presented a pulmonary aspergillosis infection which prompted a treatment with voriconazole and a reduction in immunosuppressive treatment doses: oral cyclosporine $40 \mathrm{mg}$ bid with trough level ranging from $100 \mathrm{ng} / \mathrm{ml}$ to $150 \mathrm{ng} / \mathrm{ml}$ and oral MMF $500 \mathrm{mg}$ bid. In October 2011, asymptomatic DQ5 DSA was detected with a mean fluorescence intensity (MFI) at 9695, without graft dysfunction nor pathologic sign of AMR leading to add Everolimus to the triple association of immunosuppressive treatment. In May 2012, the coronary angiogram showed moderate lesions graded CAV1 in the ISHLT classification. In June 2014, the DSA MFI level increased dramatically to 19516 associated to a dyspnea NYHA class II, bilateral legs edema, a LV dysfunction with LVEF decreasing from $70 \%$ to $40 \%$ and a pAMR2 graded EMB. The treatment associated 13 plasma exchange (EP) procedures and intravenous immunoglobulin (IVIG; Privigen ${ }^{\otimes}$ ) treatment with $0.5 \mathrm{~g} / \mathrm{kg}$ after each other EP procedures followed by a single $375 \mathrm{mg} / \mathrm{m}^{2}$ dose of Rituximab according to our local protocol. The coronary angiogram showed a grade CAV 3 with multiple lesions necessitating 5 coronary stent implantations. In January 2015, the EMB showed a pAMR1 without any decrease of DSA level nor LVEF increase. Because the absence of complete regression of AMR on repeated biopsies and the persistence of signs of graft dysfunction, Belimumab was introduced at a dose of $10 \mathrm{mg} / \mathrm{kg}$ IV at D0, D14 and D21 associated to EP and IVIG twice a week. Belimumab was well tolerated and clinical symptoms decreased. In March 2015, the patient was admitted in ICU with severe breath distress due to pulmonary pneumocystosis and Klebsiella pneumonia infections associated to lymphopenia and hypoglobulinemia treated by large spectrum antibiotics, Atovaquone, and a perfusion of $2 \mathrm{~g} / \mathrm{kg}$ IVIG after stopping EP. The patient was discharged from ICU in May 2015 and from our hospital in June 2015 with a normal LVEF. Repeated echocardiographies showed sub-normal LVEF ranging between $40 \%$ and $50 \%$ and EMB were graded pAMR0 in September and December 2015 despite a still high DSA MFI level. He died in June 2016 after stopping all his treatments due to a severe depression.

\section{Discussion}

There is no consensus for the treatment of AMR. The guidelines are based on expert recommendations and the decision to treat depends on the symptomatic and immunologic characteristics [1]. The treatment protocol is still being discussed and depends on the severity of the cardiac consequences [1].

${ }^{\star}$ Correspondence to: Eric Epailly, Cardiovascular Surgery Department, University Hospital Strasbourg, France, Tel: +33369550813; E-mail: Eric. Epailly@chru-strasbourg.fr

Key words: heart transplantation, antibody-mediated-rejection, belimumab

Received: March 15, 2019; Accepted: March 27, 2019; Published: March 29, 2019 
Two retrospective studies on HTx patients showed the beneficial effect of Rituximab on LVEF, on the complete histological remission and the significant increase of survival to 3 years without increasing the infection and rehospitalisation rates [2,3]. But its efficacy is still debated: the RITUX-ERAH study showed no benefit of Rituximab compared to placebo to treat AMR after kidney transplantation [4]. Eculizumab (antiC5) is a complement inhibitor used to treat AMR in association to plasmapheresis, but its efficacy is also still debated. For this reason and because of the high cost of Eculizumab, we did not choose this treatment option. Belimumab inhibits the BL survival and their transformation in plasmocytes by linking the BAFF soluble protein and lowering the level of circulating antibodies [5]. A randomised double blinded phase 2 study demonstrated the safety and the potential effect of Belimumab on AMR added to a standard of care treatment after renal on 25 kidney transplanted patients [6]. High BAFF levels before heart transplantation has been found to be a risk factor of acute cellular rejection [7]. There is no study demonstrating the efficacy of Belimumab on post HTx AMR. Its benefits compared to other molecules is unknown. Our protocol associating steroids, IVIG, plasmapheresis and one Rituximab injection during the 3 months preceding the use of Belimumab did not normalize EMB evidencing the persistence of AMR. Belimumab successfully ameliorated the symptoms and allowed a long-term normalization of the EMB results despite unchanged high DSA MFI levels.

\section{Conclusion}

This is the first case evidencing the efficacy of Belimumab for the treatment of AMR after heart transplantation. Because of its well tolerated original mechanism of action by inhibiting the production of plasmocytes, Belimumab could be a treatment option in HTx patients suffering from AMR. Further studies are requested to confirm its efficacy.

\section{References}

1. AHA scientific statement (2015) antibody-mediated rejection in cardiac transplantation emerging knowledge in diagnosis and management. Circulation 131: 1608-1639.

2. Ravichandran AK, Schilling JD, Novak E, Pfeifer J, Ewald GA, et al. (2013) Rituximab is associated with improved survival in cardiac allograft patients with antibodymediated rejection: a single center review. Clin Transplant 27: 961-967.

3. Garrett HE Jr, Duvall-Seaman D, Helsley B, Groshart K (2005) Treatment of vascular rejection with rituximab in cardiac transplantation. $J$ Heart LungTransplant 24: 13371342 .

4. Sautenet B, Blancho G, Buchler M, Emmanuel M, Olivier T, et al. (2013) Oneyear results of the effects of rituximab on acute humoral rejection in renal transplantation: RITUX ERAH, a multicenter randomized placebo-controlled trial [abstract]. Am J Transplant 13.

5. Lenert $\mathrm{P}$, Lenert $\mathrm{A}(2015)$ Current and emerging treatment options for ANCA-associated vasculitis: potential role of belimumab and other BAFF/APRIL targeting agents. Drug Des Devel Ther 9: 333-347.

6. Banham GD, Flint SM, Torpey N, Lyons PA, Shanahan, DN, et al. (2018) Belimumab in kidney transplantation: an experimental medicine, randomised, placebo-controlled phase 2 trial. Lancet 391: 2619-2630.

7. Sarmiento E, Jaramillo M, Calahorra L, Fernandez-Yañez J, Gomez-Sanchez M, et al. (2017) Evaluation of humoral immunity profiles to identify heart recipients at risk for development of severe infections: a multicenter prospective study. J Heart Lung Transplant 38: 529-539.

Copyright: (C2019 Ade M. This is an open-access article distributed under the terms of the Creative Commons Attribution License, which permits unrestricted use, distribution, and reproduction in any medium, provided the original author and source are credited. 